\title{
Effect of different agents onto multidrug resistant tumor cells revealed by fluorescence correlation spectroscopy
}

\author{
Céline Boutin $^{\mathrm{a}}$, Yann Roche ${ }^{\mathrm{b}}$, Christine Millot ${ }^{\mathrm{b}}$, Régis Deturche ${ }^{\mathrm{a}}$, Jérôme Plain ${ }^{\mathrm{a}}$, \\ Pierre Jeannesson $^{\mathrm{b}}$, Michel Manfait ${ }^{\mathrm{b}}$, Pascal Royer ${ }^{\mathrm{a}}$, Jean-Marc Millot ${ }^{\mathrm{b}}$ \\ and Rodolphe Jaffiol ${ }^{\text {a,* }}$ \\ ${ }^{\text {a }}$ LNIO (Laboratoire de Nanotechnologie et d'Instrumentation Optique), Institut Charles Delaunay, \\ FRE CNRS 2848, Université de Technologie de Troyes, 10010 Troyes cedex, France \\ ${ }^{\mathrm{b}}$ Unité MéDIAN (Médicament, Dynamique Intracellulaire, Architecture Nucléaire), UMR CNRS 6142, \\ Faculté de Pharmacie, Université de Reims Champagne-Ardennes, 51096 Reims cedex, France
}

\begin{abstract}
Fluorescence correlation spectroscopy (FCS) has been used to analyze the plasma membrane fluidity and heterogeneity of multidrug resistant cells. At the single cell level, the effects of different membrane agents present in the extra-cellular medium have been explored. Firstly, we reveal a modification of plasma membrane heterogeneities according to the addition of a fluidity modulator, benzyl alcohol. On the other hand, revertants such as verapamil and cyclosporin-A appear to act more specifically on the slow diffusion sites, such as lipids microdomains.
\end{abstract}

Keywords: Fluorescence correlation spectroscopy, membrane dynamic, multidrug resistant (MDR) cells

\section{Introduction}

MultiDrug Resistance (MDR) to chemotherapeutic treatment of cancers is mainly dependent on biomechanism occurring at the plasma membrane level which involving fluidity, permeability, presence of microdomains (lipid rafts, cavealae) [1,2]. This resistance also depends on the over expression of membrane transporters such as the P-glycoprotein and a decreasing of the intracytoplasmic concentration of lipophilic cytotoxic agents [3]. All these membrane mechanisms can be monitored through the lateral diffusion of a fluorescent membrane probe, with a well-suited relevant technique: fluorescence correlation spectroscopy [4]. We have compared the plasma membrane diffusion of sensitive and MDR cells (LR73 ovarian carcinoma cells). Then we have studied the effects of different membrane agents onto MDR cells.

\footnotetext{
${ }^{*}$ Corresponding author: R. Jaffiol, LNIO (Laboratoire de Nanotechnologie et d'Instrumentation Optique), Institut Charles Delaunay, FRE CNRS 2848, Université de Technologie de Troyes, 12 rue Marie Curie, BP2060, 10010 Troyes cedex, France. Tel.: +33 3 25718527; Fax: +33 3 25718456; E-mail: rodolphe.jaffiol@utt.fr.
} 


\section{Materials and methods}

In FCS, fluctuations of the fluorescence signal are monitored while molecules are diffusing through the observation volume, which is excited in a diffraction limited illumination spot of a focused laser beam. Interestingly, significant amplitude fluctuations only arise from sufficiently low molecule concentrations, typically at the nanomolar concentration scale. These required dilution conditions are a real advantage, especially for in vivo investigations, since FCS creates only slight perturbations in living cells. Moreover, the potential of FCS to relate heterogeneity in model and natural membranes was recently demonstrated [5].

Our FCS setup is based on a conventional inverted confocal microscope, designed to probe a very small observation volume $\left(V \approx 0.35 \mu \mathrm{m}^{3}\right)$ [6]. A highly dilute solution of dyes is illuminates with a focused laser beam [4]. The fluorescence signal $F(t)$ is recorded with an avalanche photodiode, and the fluorescence fluctuations are analyzed through the temporal autocorrelation function of $F(t)$, namely the autocorrelation function (ACF) denoted $G(\tau)$. FCS experiments on plasma membrane are performed according to a fine achievement of the axial positioning of the observation volume by selecting the fastest value of the lateral diffusion-time of dyes (denoted $\tau_{d}^{2 \mathrm{D}}$ ), and simultaneously the maximum fluorescence signal $F(t)$ and its fluctuations [7]. The amphiphile fluorescent dye used (4Di-10ASP) is a specific membrane probe, well known to strongly bind to mimetic membrane systems such as lipid bilayer [8].

\section{Results and discussion}

\subsection{Data analysis}

Membrane autocorrelation functions $G(\tau)$ are fitted with the following model describing the simultaneous diffusions of both a slow (2D) and a fast (3D) components, according to the molecules diffusing in the plasma membrane and the residual contribution of dyes freely diffusing in the vicinity of the membrane, respectively:

$$
\begin{aligned}
G(\tau)=1 & +\left\{1+\frac{T_{\mathrm{eq}} \exp ^{-\tau / \tau_{\mathrm{T}}}}{1-T_{\mathrm{eq}}}\right\} \times\left\{\frac{\left\langle c r^{2 \mathrm{D}}\right\rangle^{2}}{\left\langle c r^{\text {total }}\right\rangle^{2}} \frac{1}{\left\langle N^{2 \mathrm{D}}\right\rangle}\left(1+\frac{\tau}{\tau_{d}^{2 \mathrm{D}}}\right)^{-1}\right. \\
& \left.+\frac{\left(\left\langle c r^{\text {total }}\right\rangle-\left\langle c r^{2 \mathrm{D}}\right\rangle\right)^{2}}{\left\langle c r^{\text {total }}\right\rangle^{2}} \frac{1}{\left\langle N^{3 \mathrm{D}}\right\rangle}\left(1+\frac{\tau}{\tau_{d}^{3 \mathrm{D}}}\right)^{-1}\left(1+\frac{\tau}{S^{2} \tau_{d}^{3 \mathrm{D}}}\right)^{-1 / 2}\right\},
\end{aligned}
$$

where $N^{2 \mathrm{D}}$ and $N^{3 \mathrm{D}}$ are the mean number of diffusing molecules for each component. $c r^{\text {total }}$ is the average fluorescence signal recorded (i.e. the average count-rate), whereas $c r^{2 \mathrm{D}}$ is the fluorescence contribution only for molecules diffusing in the membrane. The respective diffusion times are $\tau_{d}^{3 \mathrm{D}}$ and $\tau_{d}^{2 \mathrm{D}}$, and $S$ is the axial elongation of the observation volume ( $S \approx 5$ ). The first term of Eq. (1) describe the triplet dynamics ( $\tau_{\mathrm{T}}$ is the triplet lifetime and $T_{\mathrm{eq}}$ the fraction of molecules in the triplet state).

For each type of cell-populations studied (MDR cells, sensitive cells, ...), a statistical analysis of the diffusion time $\tau_{d}^{2 \mathrm{D}}$ (measured at the single cell level) have been performed [9]. Three different significant informations have been extracted from the diffusion-time distribution analysis: the average value, its standard deviation which gives information about population heterogeneity, and the percentage of diffusion time measurements above a threshold value, denoted $\tau_{S}$, that points out slow diffusion sites 


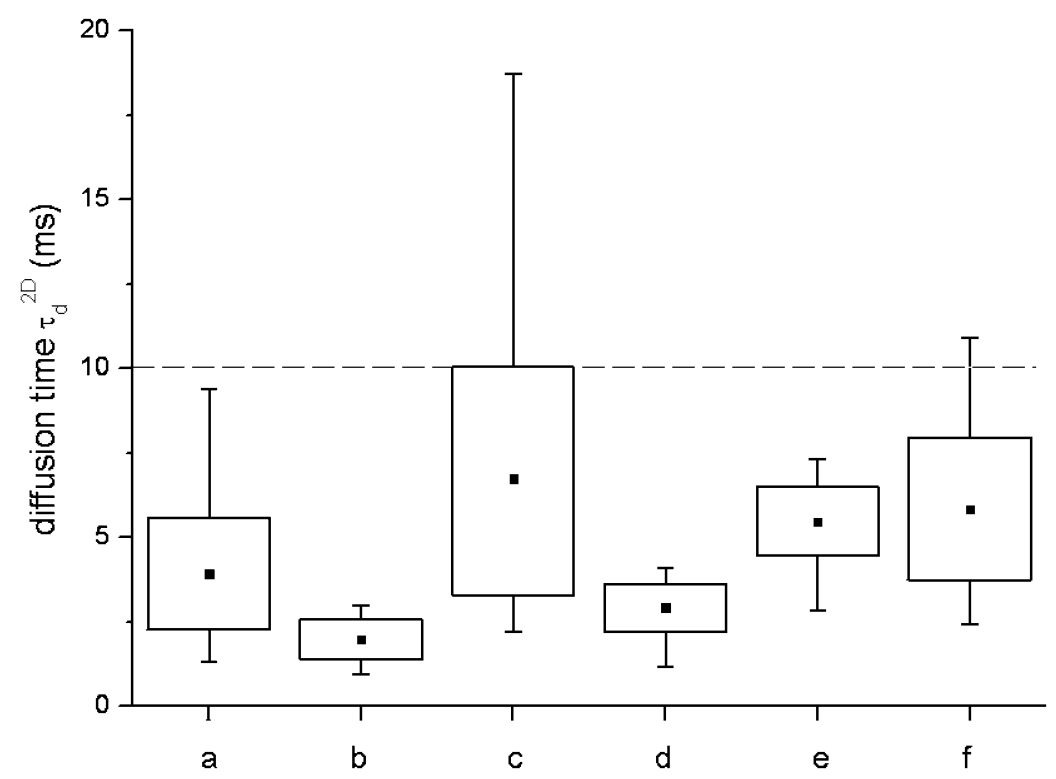

Fig. 1. Box chart representation of the diffusion times for (a) sensitive cells, (b) sensitive cells with benzyl alcohol, (c) MDR cells, (d) MDR cells with benzyl alcohol, (e) MDR cells with verapamil and (f) MDR cells with cyclosporine-A. - the average value, "square box" - the standard deviation and $T$ - the distribution of measurements (min-max).

such as lipids microdomains or rafts. $\tau_{S}$ has been defined as the highest $\tau_{d}^{2 \mathrm{D}}$ value obtained for sensitive cells and has been fixed to $10 \mathrm{~ms}$.

\subsection{Comparison of the plasma membrane fluidity between sensitive and MDR cells}

Characterization of the MDR and sensitive cell-lines has been conducted on a large population of cells (Fig. 1(a and c)). Both distributions are large and point out the heterogeneity of the plasma membrane. The distribution of the diffusion times for MDR cells is larger than for sensitive cells as indicated by the standard deviation. Consequently, the plasma membrane of MDR cells appears to be more heterogeneous. Indeed $20 \%$ of the measured $\tau_{d}^{2 \mathrm{D}}$ are higher than $\tau_{S}=10 \mathrm{~ms}$ and then correspond to slow diffusion sites (specific microdomains for example).

These differences can be related to the lipid composition of the plasma membrane. Membrane fatty acid composition shows that MDR cells contain a high proportion of saturated fatty acid in comparison to sensitive cells [10]. Saturated fatty acids are known to increase the membrane microviscosity and are mainly localized in organized microdomains (such as rafts) [11]. That explains to a certain extant the differences between the diffusion-times distributions of sensitives and resistant cells.

\subsection{Effects of modulators on the plasma membrane fluidity}

Figure 2 clearly shows the fluidizing effect of the benzyl alcohol on the membrane diffusion of MDR cells. The addition of benzyl alcohol induces a global significant increase of the plasma membrane fluidity and reduces considerably its heterogeneity tending towards phospholipids vesicle behavior (i.e. lateral diffusion of a pure lipid bilayer membrane), data not presented.

Contrarily to the benzyl alcohol, which is well known as membrane fluidizer [2], we have proved that revertant agents such as verapamil and cyclosporine-A do not show a fingerprint of fluidity similar 


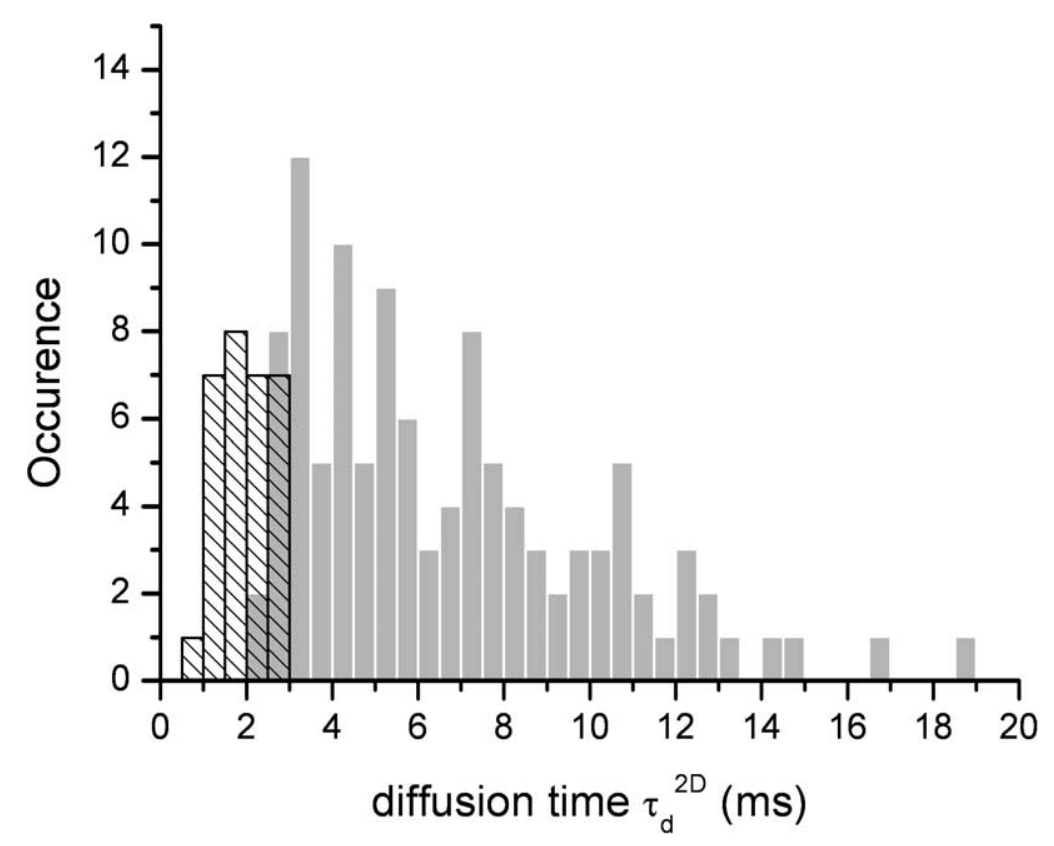

Fig. 2. Distributions of membrane diffusion times obtained for two populations of MDR cells before (gray columns) and after (striped columns) the addition of benzyl alcohol. Column bin size is $0.5 \mathrm{~ms}$.

to that observed with benzyl alcohol (Fig. 1). In fact, they significantly reduce the percentage of slow diffusion sites (above $\tau_{S}=10 \mathrm{~ms}$ ). A possible explanation is that while benzyl alcohol induces a global fluidizing effect on the plasma membrane, revertant agents seem to act more precisely on slow diffusion sites, such as microdomains. The multidrug resistance phenotype is characterized by the presence of the P-glycoprotein. Previous studies have suggested that a significant fraction of this protein is located in raft domains [12]. Furthermore, P-glycoprotein is a lipid translocase that handles the lipids constituting its direct environment (the lipid rafts) [13]. The inhibition of the P-glycoprotein by revertant agents could increase the membrane fluidity by disturbing the direct environment of this protein.

\section{Conclusion}

Through the lateral diffusion of a fluorescent dye, we point out the plasma membrane microviscosity signature of MDR cells. The broad distribution of the diffusion times $\tau_{d}^{2 \mathrm{D}}$ recorded on MDR cells can be obviously related to the high heterogeneity of such plasma membrane (high heterogeneity in term of composition and organization). The presence of distinct slow diffusion sites (i.e. specific microdomains) linked to the resistance has been highlighted through the action of revertant agents.

\section{Acknowledgment}

We authors thank the Région Champagne-Ardenne (France) for financial support under the contract called "Projet émergence: Spectroscopie de corrélation de fluorescence en champ proche optique: applications aux biomolécules et biomatériaux". 


\section{References}

[1] V.A. Oleinikov, F. Fleury, A. Ianoul, S. Zaitsev and I. Nabiev, P-glycoprotein effect on the properties of its natural lipid environment probed by Raman spectroscopy and Langmuir-Blodgett technique, FEBS Lett. 20 (2006), 4953-4958.

[2] R. Regev, Y.G. Assaraf and G.D. Eytan, Membrane fluidization by ether, other anesthetics, and certain agents abolishes P-glycoprotein ATPase activity and modulates efflux from multidrug-resistant cells, Eur. J. Biochem. 259 (1999), 18-24.

[3] J.D. Allen, R.F. Brinkhuis, L. van Deemter, J. Wijnholds and A.H. Schinkel, Extensive contribution of the Multidrug Transporters P-glycoprotein an Mrp1 to basal drug resistance, Cancer Res. 60 (2000), 5761-5766.

[4] M. Gosch and R. Rigler, Fluorescence correlation spectroscopy of molecular motions and kinetics, Adv. Drug. Deliv. Rev. 1 (2005), 169-190.

[5] E. Haustein and P. Schwille, Ultrasensitive investigations of biological systems by fluorescence correlation spectroscopy, Methods 2 (2003), 153-166.

[6] C. Boutin, R. Jaffiol, J. Plain and P. Royer, Influence of the surface hydrophobicity on fluorescence correlation spectroscopy measurements, in: Progress in Biomedical Optics and Imaging, Proc. SPIE, Vol. 6444, 2007, p. 64440J.

[7] J. Humpolickova, E. Gielen, A. Benda, V. Fagulova, J. Vercammen, M. Vandeven, M. Hof, M. Ameloot and Y. Engelborghs, Probing diffusion laws within cellular membranes by Z-scan fluorescence correlation spectroscopy, Biophys. J. 91 (2006), 23-25.

[8] L.M. Loew and L.L. Simpson, Charge-shift probes of membrane potential. A probable electrochromic mechanism for P-aminostyrylpyridinium probes on a hemispherical lipid bilayer, Biophys. J. 34 (1981), 353-365.

[9] H. Jankevics, M. Prummer, P. Izewska, H. Pick, K. Leufgen and H. Vogel, Diffusion-time distribution analysis reveals characteristic ligand-dependent interaction patterns of nuclear receptors in living cells, Biochemistry 44 (2005), 1167611683.

[10] M.T. Santini, R. Romano, G. Rainaldi, P. Filippini, E. Bravo, L. Porcu, A. Motta, A. Calcabrini, S. Meschini, P.L. Indovina and G. Arancia, The relationship between 1H-NMR mobile lipid intensity and cholesterol in two human tumor multidrug resistant cell lines (MCF-7 and LoVo), Biochim. Biophys. Acta 30 (2001), 111-131.

[11] K. Simons and E. Ikonen, Functional rafts in cell membranes, Nature 5 (1997), 569-572.

[12] Z. Bacso, H. Nagy, K. Goda, L. Bene, F. Fenyvesi, J. Matko and G. Szabo, Raft and cytoskeleton associations of an ABC transporter P-glycoprotein, Cytometry A 61 (2004), 105-116.

[13] S. Orlowski, S. Martin and A. Escargueil, P-glycoprotein and 'lipid rafts': some ambiguous mutual relationships (floating on them, building them or meeting them by chance?), Cell. Mol. Life Sci. 63 (2006), 1038-1059. 


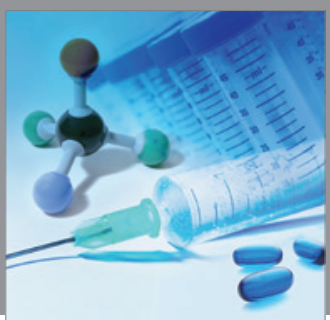

International Journal of

Medicinal Chemistry

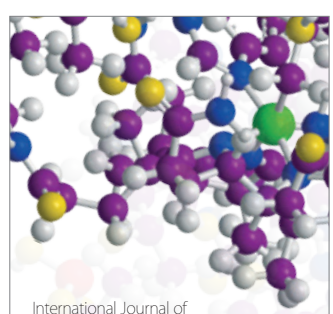

Carbohydrate Chemistry

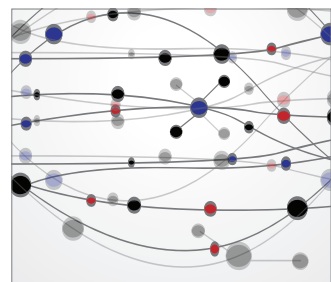

The Scientific World Journal
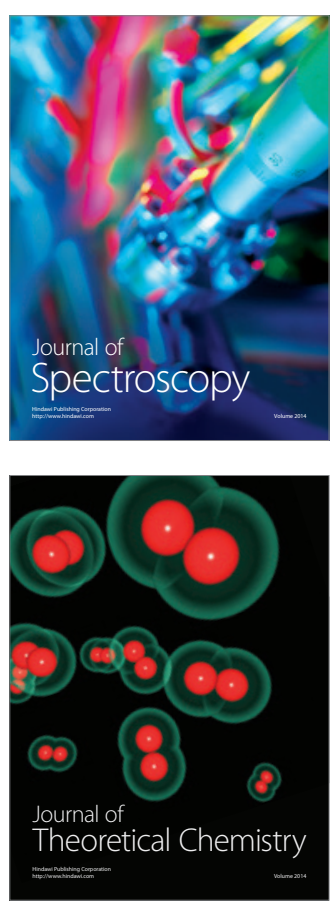
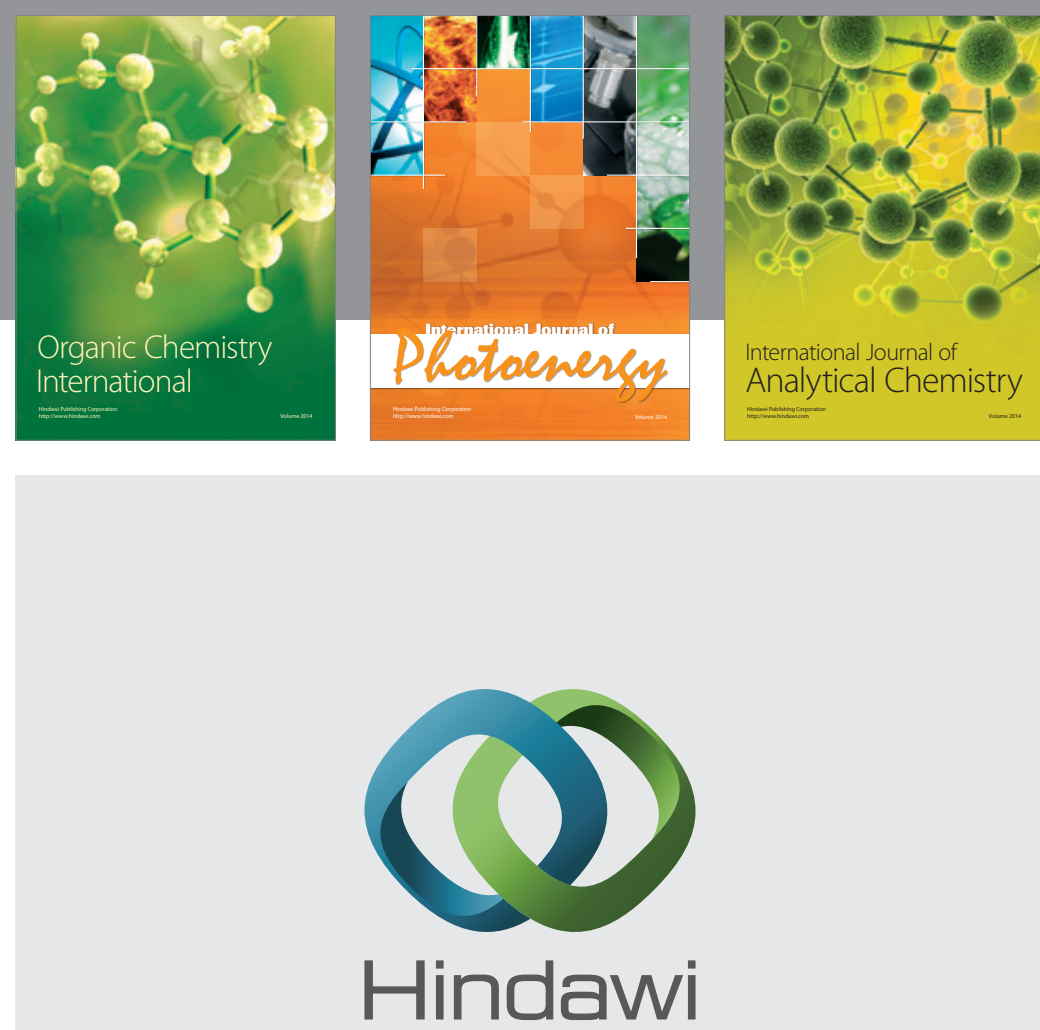

Submit your manuscripts at

http://www.hindawi.com
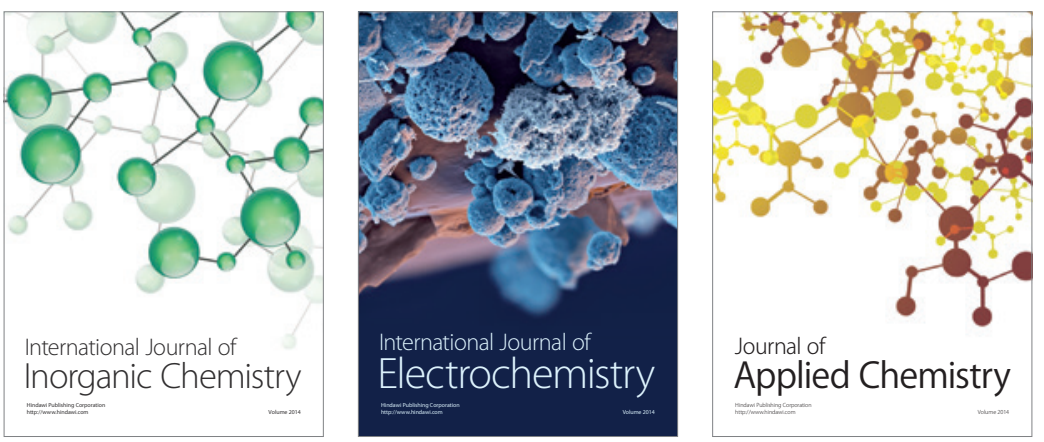

Journal of

Applied Chemistry
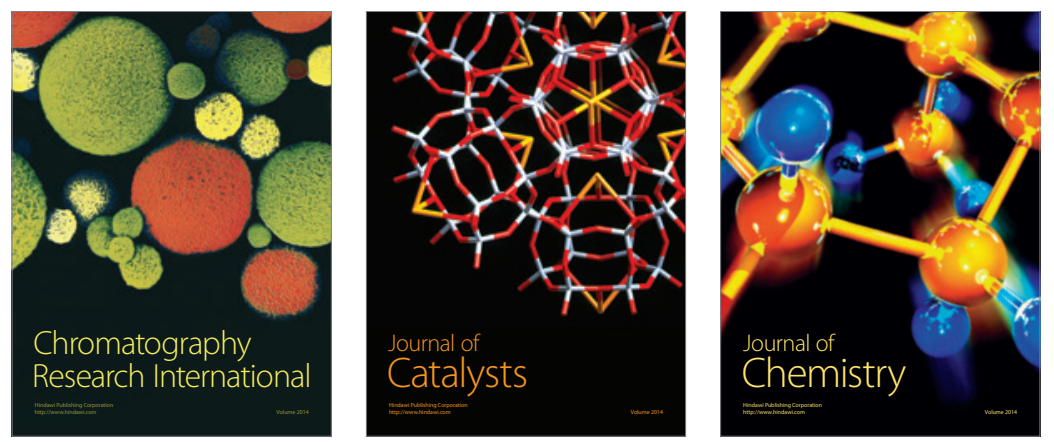
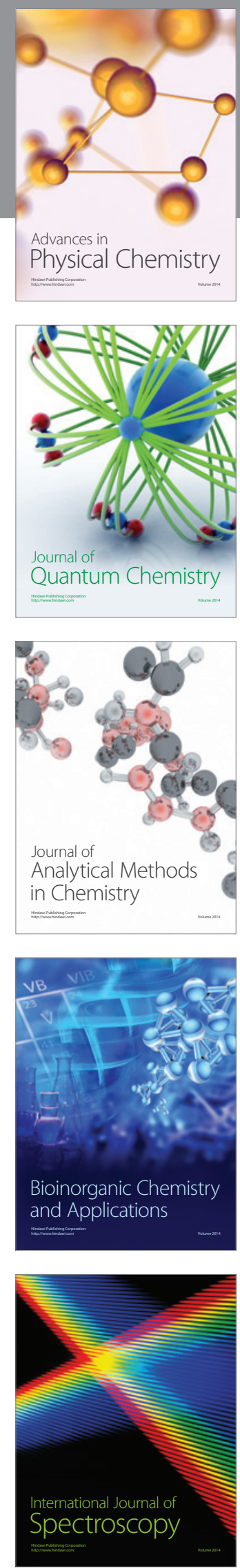